\title{
Stability conditions for the solutions of the half-projected Hartree-Fock scheme. The lithium-hydride ground state
}

\author{
Yves Gabriel Smeyers and Gerardo Delgado-Barrio \\ Instituto de Estructura de la Materia del Consejo Superior de Investigaciones Cientificas, Serrano 119, Madrid-6, Spain \\ (Received 7 September 1976)
}

\begin{abstract}
The stability conditions for the solutions of a two-unrestricted-determinant function (the half-projected Hartree-Fock function) are deduced from the necessary requirements for the minimization of the energy functional. The theory is applied to the case of the $\mathrm{LiH}$ ground state, in order to investigate the various solutions encountered in the variational problem. It is found that the two lowest solutions are local minima. The number of these solutions is estimated and their significance discussed as a function of the nuclear separation.
\end{abstract}

\section{INTRODUCTION}

The stability conditions for the solutions of the Hartree-Fock equations were first formulated by Thouless for a single determinant function. ${ }^{1}$ This author deduced the expression that ensures the minimization of the wave function from the necessary conditions for minimizing the energy functional. Adams reformulated the stability conditions from the first-order density-matrix formalism and compared the relative stability of the restricted (RHF) and unrestricted Hartree-Fock schemes (UHF). ${ }^{2}$ Similarly, Cizek and Paldus studied the instability of the RHF solutions with respect to variations, which release the double occupancy conditions, in cyclic polyenes, linear polyacenes, and odd linear polyenic radicals. ${ }^{3}$ Furthermore, from the variational conditions, the multiple solutions to the RHF problem were investigated by Stanton. ${ }^{4}$ On the other hand, the stability of a RHF function with respect to symmetry breakdowns was studied by Kaplan et al. from another point of view based on an upperbound theorem for the RHF ground energy. ${ }^{5}$

In the present work, we extend the above investigations and deduce the stability conditions for the solutions of a two-unrestricted-determinant function, i.e., the half-projected HartreeFock function (HPHF). ${ }^{6}$ The latter has been found to be very useful in the study of bond-breaking process, ${ }^{7}$ and was successfully employed in calculations on small molecules. ${ }^{8}$

As is well known, the HPHF function has the form

$$
\begin{aligned}
\psi^{\mathrm{HPHF}}= & \hat{A}(S) \psi^{\mathrm{UHF}} \\
= & \frac{1}{2}\left[\left|\varphi_{1} \bar{\varphi}_{1}^{\prime} \varphi_{2} \bar{\varphi}_{2}^{\prime} \cdots\right|\right. \\
& \left.+(-1)^{n+S}\left|\bar{\varphi}_{1} \varphi_{1}^{\prime} \bar{\varphi}_{2} \varphi_{2}^{\prime} \cdots\right|\right],
\end{aligned}
$$

where $\hat{A}(S)$ is the half-projection operator which projects on the subspace of quantum numbers $S$ even or odd. ${ }^{6,7}$

When $S$ is even, the HPHF function may be successfully used for the study of singlet ground states, because it does not contain triplet contaminants. In fact, the HPHF function in this case only has one shell which is clearly split. Therefore this function is shown to be approximately equivalent to two natural configurations of closed shells: the fundamental and a second doubly excited one. ${ }^{9}$

Some difficulties arise in the determination of the HPHF function because various solutions, lower than the RHF one, are often encountered in the variational procedure. ${ }^{8,9}$ In this work, we examine this problem from the stability point of view. For this purpose we have investigated the two lowest solutions, found for the $\mathrm{LiH}$ ground state, as a function of the nuclear separation.

\section{HPHF STABILITY CONDITIONS}

A way for determining if the HPHF solution corresponds to a minimum (or a saddle point) is to study the second variation of the energy functional. If this second variation is positive definite the solution corresponds to a real minimum. This may be, however, of local type because of the range of the variation.

Let us express the variation of the occupied orbitals $\varphi_{p}$ and $\varphi_{p}^{\prime}$ in terms of the virtual ones. Thus,

$$
\delta \varphi_{p}=\sum_{t} c_{p}^{t} \varphi_{t}, \quad \delta \varphi_{p}^{\prime}=\sum_{t} d_{p}^{t} \varphi_{t}^{\prime},
$$

where the coefficients $c_{p}^{t}$ and $d_{p}^{t}$ are infinitesimal. The first variation of the HPHF function may then be written as a linear combination of singly ex- 
cited HPHF functions between primed and unprimed orbitals:

$$
\delta \psi=\sum_{p, t}\left[c_{p}^{t} \psi_{t}^{p}+d_{p}^{t} \psi_{t^{\prime}}^{p^{\prime}}\right] .
$$

These singly excited HPHF functions must, however, be renormalized in order to properly construct the matrix elements corresponding to the second variation of the energy.

The second variation of the wave function may be written likewise in terms of doubly excited HPHF functions:

$$
\delta^{2} \psi=\sum_{p t} \sum_{q v}\left[c_{p}^{t} c_{q}^{v} \psi_{t v}^{p q}+c_{p}^{t} d_{q}^{v} \psi_{t v^{\prime}}^{p q^{\prime}}+\cdots\right]
$$

As is well known, the second variation of the mean energy can be written

$\langle\psi \psi\rangle \delta^{2} E=\left\langle\delta^{2} \psi H \psi\right\rangle+2\langle\delta \psi(H-E) \delta \psi\rangle+\left\langle\psi H \delta^{2} \psi\right\rangle$.

Substituting (3) and (4) into (5) one obtains

$$
\begin{aligned}
\langle\psi \psi\rangle \delta^{2} E= & \sum_{p t} \sum_{q v}\left[c_{p}^{* t} c_{q}^{* v}\left\langle\psi_{t v}^{p q} H \psi\right\rangle+2 c_{p}^{* t} d_{q}^{* v}\left\langle\psi_{t v^{\prime}}^{p q^{\prime}} H \psi\right\rangle+d_{p}^{* t} d_{q}^{* v}\left\langle\psi_{t^{\prime} v^{\prime}}^{p^{\prime} q^{\prime}} H \psi\right\rangle\right] \\
& +\sum_{p t} \sum_{q v} 2\left[c_{p}^{* t} c_{q}^{v}\left\langle\psi_{t}^{p}(H-E) \psi_{v}^{q}\right\rangle+c_{p}^{* t} d_{q}^{v}\left\langle\psi_{t}^{p}(H-E) \psi_{v^{\prime}}^{q^{\prime}}\right\rangle+d_{p}^{* t} c_{q}^{v}\left\langle\psi_{t^{\prime}}^{p^{\prime}}(H-E) \psi_{v}^{q}\right\rangle+d_{p}^{* t} d_{q}^{v}\left\langle\psi_{t^{\prime}}^{p^{\prime}}(H-E) \psi_{v^{\prime}}^{q^{\prime}}\right\rangle\right] \\
& +\sum_{p t} \sum_{q v}\left[c_{p}^{t} c_{q}^{v}\left\langle\psi H \psi_{t v}^{p q}\right\rangle+c_{p}^{t} d_{q}^{v}\left\langle\psi H \psi_{t v^{\prime}}^{p q^{\prime}}\right\rangle+d_{p}^{t} d_{q}^{v}\left\langle\psi H \psi_{t^{\prime} v^{\prime}}^{p^{\prime} q^{\prime}}\right\rangle\right] .
\end{aligned}
$$

This expression has a quadratic form in the coefficients $c_{p}^{t}$ and $d_{p}^{t}$ and its Hermitian matrix may be written in the usual way:

$$
\left(\begin{array}{cc}
A & B \\
B^{*} & A^{*}
\end{array}\right),
$$

where $A$ and $B$ are likewise $2 \times 2$ submatrices depending on the type of orbitals (primed or unprimed) involved in the excitation. If the matrix (7) is positive definite the HPHF wave function will correspond to an actual minimum.

Expression (7) was derived under the assumption that $A$ and $B$ may be complex. The orbitals are, however, usually developed in terms of real coefficients. Thus $A$ and $B$ should be made up of real numbers. Accordingly, the matrix (7) may be rewritten after partial diagonalization in the following manner:

$$
\left(\begin{array}{cc}
A+B & 0 \\
0 & A-B
\end{array}\right),
$$

where the submatrices $A+B$ and $A-B$ correspond to variations in the real and imaginary space, respectively.

\section{APPLICATION TO THE LiH GROUND STATE}

In this section, the theory of stability is applied to the case of LiH ground state, in which two solutions lower than the RHF one were encountered for all the nuclear configurations considered.

In Ref. 7, the lowest solution was taken as the correct one, in order to study the performances of the HPHF scheme. A very satisfactory po- tential-energy curve was obtained. This choice yields, however, some discontinuity in the properties of the wave function when drawn as a function of nuclear separation. This discontinuity occurs because the nature of the excited configuration, which appears in the configurational equivalent, changes in the region of bond breaking. ${ }^{9}$

Now, if the two lowest solutions were considered, it would seem more reasonable to classify them according to the nature of this excited configuration, that is, according to the type of shell which is being split. In this way, two potential-energy curves, which cross, are obtained (see Fig. 1). The problem consists then of verifying whether these two solutions are connected in some way between themselves, i.t., whether the solution corresponding to the higher-energy value is either a saddle point or a local minimum.

In order to study the stability of these two HPHF solutions we resorted to expression (8), since the HPHF orbitals were restricted to the real space. The matrix elements of such an expression were obtained, after renormalization of the excited configurations, from the calculations of the HPHF wave function. For these calculations a procedure based on the generalized Brillouin's theorem was used. $^{6}$

For simplification, the HPHF functions were recalculated using a very limited basis set, since we are more interested in the behavior of the solutions than in the numerical values. In fact, the truncation would essentially affect the position of the crossing point. This basis set was, thus, restricted to only four STO's and these were the same for both solutions. 


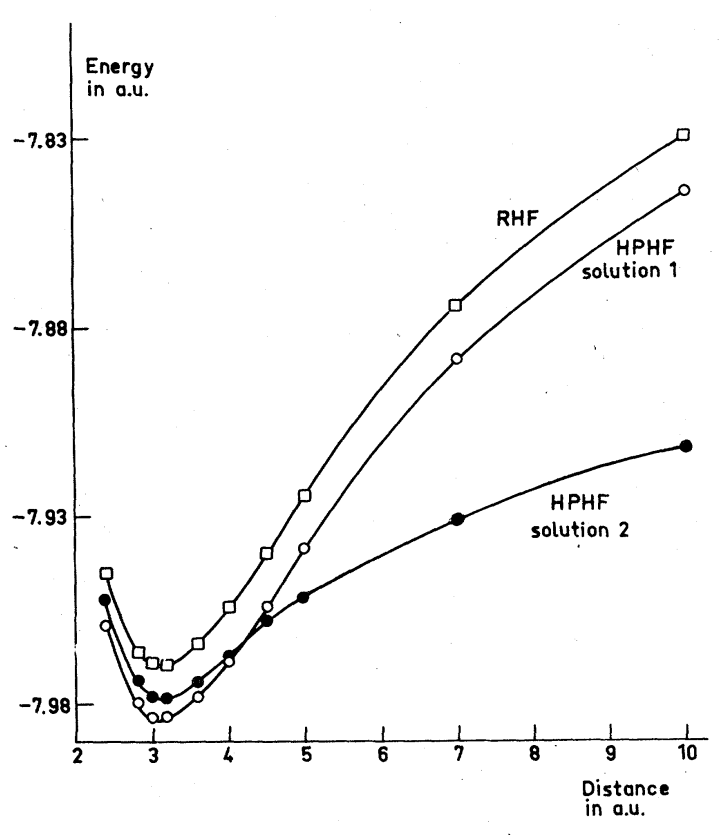

FIG. 1. Energy values vs the nuclear separation for two HPHF solutions, classified according to the nature of their split shell, together with the RHF ones.

The energy values encountered in such a way for both solutions, as a function of the internuclear distance $R$, are given in Table I, together with the basis set employed. Solution 1, which has its inner shell split, yields better energy values in the equilibrium region. Solution 2, which has its outer shell open, gives on the contrary better results at distances larger than 4 a.u., and is capable of describing correctly the breakup of the molecule with $R$. These results are summarized in Fig. 1, where the RHF potential-energy curve calculated in the same basis is included for comparison.
After diagonalization of the quadratic form (8), it is seen that this form is positive definite in the case of both solutions and for all the nuclear separations considered. It may be thus concluded that both solutions are stable solutions corresponding to two nonconnected local minima. These two minima correspond to different physical situations. Solution 1 introduces correlation effects in the $K$ shell of the $\mathrm{Li}$ atom, whereas solution 2 in the valence shell of the LiH molecule. In the approximation considered here, it is not possible to go over from one minimum to the other without introducing drastic changes in the wave function.

In Table II, the lowest eigenvalues corresponding to the submatrices $(A+B)$ and $(A-B)$ for both solutions as functions of the internuclear separation are collected. They are all positive. It appears they are maximum in the equilibrium region. It is seen, however, that the eigenvalue, corresponding to a split inner shell and a variation in the imaginary space (matrix $A-B$ ), obviously decreases with the internuclear distance. This feature suggests that, at very large distances, solution 1 could become a saddle point connected with a lower-complex solution.

\section{DISCUSSION}

In the present work, the stability conditions for a two-unrestricted-determinant function are deduced. These conditions have the habitual form of a matrix. The elements of this matrix are built firstly between all the possible singly excited configurations (matrix $A$ ), and secondly from all the possible doubly excited configurations with the fundamental one (matrix $B$ ). The procedure becomes obviously rapidly unmanageable as the number of electrons or basis functions increases. The procedure was applied to a very

TABLE I. Energy values versus the nuclear separation $R$ encountered for the two lowest solutions corresponding to $\mathrm{LiH}$ ground states, in a.u., together with the STO's employed.

\begin{tabular}{lcccccc}
\hline \hline & \multicolumn{3}{c}{ STO exponents } & \multicolumn{2}{c}{ Energy } \\
\multicolumn{1}{c}{$1 s_{\mathrm{Li}}$} & $1 s_{\mathrm{Li}}^{\prime}$ & $2 s_{\mathrm{Li}}$ & $1 s_{\mathrm{H}}$ & Solution 1 & Solution 2 \\
\hline 2.4 & 3.70 & 2.25 & 0.74 & 0.88 & -7.95918 & -7.95248 \\
2.8 & 3.70 & 2.25 & 0.79 & 0.87 & -7.98031 & -7.97453 \\
3.0157 & 3.70 & 2.25 & 0.80 & 0.86 & -7.98363 & -7.97826 \\
3.2 & 3.70 & 2.25 & 0.80 & 0.85 & -7.98368 & -7.97868 \\
3.6 & 3.70 & 2.25 & 0.78 & 0.84 & -7.97824 & -7.97506 \\
4.0 & 3.70 & 2.25 & 0.76 & 0.83 & -7.96857 & -7.96748 \\
4.5 & 3.72 & 2.26 & 0.78 & 0.88 & -7.95087 & -7.95872 \\
5.0 & 3.74 & 2.27 & 0.75 & 0.89 & -7.92929 & -7.95161 \\
7.0 & 3.74 & 2.28 & 0.70 & 0.90 & -7.87791 & -7.93086 \\
10.0 & 3.74 & 2.29 & 0.65 & 0.80 & -7.84329 & -7.91153 \\
\hline \hline
\end{tabular}


TABLE II. Lowest eigenvalues of the $A+B$ and $A-B$ matrices versus internuclear distance $R$, in a.u., for two solutions corresponding to the $\mathrm{LiH}$ ground state.

\begin{tabular}{|c|c|c|c|c|}
\hline \multirow[b]{2}{*}{$R$} & \multicolumn{2}{|c|}{ Solution 1} & \multicolumn{2}{|c|}{ Solution 2} \\
\hline & $A+B$ & $A-B$ & $A+B$ & $A-B$ \\
\hline 2.4 & 0.485 & 0.374 & 0.144 & 0.144 \\
\hline 2.8 & 0.500 & 0.383 & 0.153 & 0.153 \\
\hline 3.0157 & 0,501 & 0.377 & 0.153 & 0.154 \\
\hline 3.2 & 0.497 & 0.366 & 0.152 & 0.152 \\
\hline 3.6 & 0.477 & 0.336 & 0.145 & 0.146 \\
\hline 4.0 & 0.451 & 0.304 & 0.136 & 0.136 \\
\hline 4.5 & 0.464 & 0.286 & 0.141 & 0.141 \\
\hline 5.0 & 0.475 & 0.264 & 0.152 & 0.152 \\
\hline 7.0 & 0.395 & 0.166 & 0.136 & 0.136 \\
\hline 10.0 & 0.303 & 0.098 & 0.118 & 0.118 \\
\hline
\end{tabular}

simple case: the HPHF solutions obtained with four STO's for the LiH ground state. It is found that the two lowest solutions are, in this case, stable solutions, i.e., that both correspond to local minima.

The next question arising is to what extent these results may be extrapolated to molecular systems of increasing complexity. In this sense, one may anticipate that the number of possible stable so- lutions will be given by the number of different physical situations, that is, the number of shells in the wave function under consideration.

All these HPHF solutions may be regarded, of course, as restricted forms of a more general configuration interaction function. Therefore, the significance of the different HPHF solutions will vary according to their applications. For example, in the case of the $\mathrm{LiH}$ ground state the solution 2 will give better results with respect to properties depending essentially on the valence shell, like force constants, dissociation, etc. On the other hand, solution 1 will work better for properties depending on the inner shell. Thus, for properties depending on both shells, like the total energy, solution 1 will be better in the equilibrium region whereas solution 2 will be better at distance larger than 4.0 a.u.

The existence of various solutions in the variational problem is not restricted to the HPHF model. Spurious solutions were previously found in the UHF model ${ }^{10}$ and full projected HartreeFock scheme (PHF). ${ }^{11}$ Since the PHF function seems to behave like the HPHF function, the conclusions presented here, would be valid for the full projected case. ${ }^{9}$ A study of this problem is now in progress in our laboratory.
${ }^{1} \mathrm{D}$. J. Thouless, The Quantum Mechanics in Many Body Systems (Academic, New York, 1961).

${ }^{2}$ W. H. Adams, Phys. Rev. 127, 1650 (1962).

${ }^{3} \mathrm{~J}$. Cizek and J. Paldus, J. Chem. Phys. 47, 3976 (1967); 53, 821 (1970); Phys. Rev. A $\underline{3}, 525$ (1971); J. Paldus and J. Cizek, J. Chem. Phys. $\underline{52}, 2919$ (1970); 54, 2293 (1971); Phys. Rev. A 2,2268 (1970).

${ }^{4}$ R. E. Stanton, J. Chem. Phys. 48, 257 (1968).

${ }^{5}$ T. A. Kaplan and W. H. Kleiner, Phys. Rev. 156, 1 (1967).

${ }^{6}$ Y. G. Smeyers, An. Fis. (Madrid) 67, 17 (1971); Y. G. Smeyers and L. Doreste-Suarez, Int. J. Quantum
Chem. 7, 687 (1973).

${ }^{7}$ Y. G. Smeyers and G. Delgado-Barrio, Int. J. Quantum Chem. 8 , 733 (1974); An. Fis. (Madrid) 71, 50 (1975).

${ }^{8}$ P. A. Cox and M. H. Wood, Theor. Chim. Acta (Berlin) 41, 269 (1976); 니, 279 (1976).

${ }^{9}$ Y. G. Smeyers and G. Delgado-Barrio, Int. J. Quantum Chem. 10, 461 (1976).

${ }^{10}$ R. F. Prat, Phys. Rev. A $\underline{6}, 1735$ (1972); G. DelgadoBarrio and R. F. Prat, Phys. Rev. A 12, 2288 (1975).

${ }^{11}$ R. Lefebvre and Y. G. Smeyers, Int. J. Quantum Chem. 1, 441 (1967). 